\title{
Ability of Stratus OCT to Identify Localized Retinal Nerve Fiber Layer Defects in Patients with Normal Standard Automated Perimetry Results
}

\author{
Tae-Woo Kim, ${ }^{1,2}$ Un-Chul Park, ${ }^{1}$ Ki Ho Park, ${ }^{1}$ and Dong Myung Kim ${ }^{1}$
}

Purpose. To evaluate the ability of Stratus optical coherence tomography (OCT; Carl Zeiss Meditec, Dublin, CA) with its internal normative database to discriminate between healthy eyes and eyes with a localized retinal nerve fiber layer (RNFL) defect not accompanied by a perimetric defect according to standard automated perimetry (SAP) (i.e., a preperimetric localized RNFL defect).

Methods. Data were retrospectively reviewed from 49 eyes of 49 subjects with preperimetric localized RNFL defects, and 49 healthy control eyes of 49 age-matched subjects, with classification into each group based primarily on red-free fundus photography results. The sensitivity and specificity of various OCT RNFL thickness parameters for identifying subjects with preperimetric localized RNFL defects was determined. In addition, the topographic relationship between the OCT-measured RNFL thickness and the localized RNFL defect location according to red-free fundus photography (i.e., the red-free RNFL defect) was examined.

Results. The sensitivity of the Stratus OCT RNFL thickness parameters ranged from $2.0 \%$ to $40.8 \%$. The highest sensitivity was yielded using a criterion of $\geq 1$ clock hour that were abnormal at the $5 \%$ level, which had a specificity of $85.7 \%$. The OCT-measured RNFL thickness showed excellent topographic agreement with the red-free RNFL defect location.

Conclusions. Although the sensitivity of current Stratus OCT RNFL thickness parameters is low for preperimetric localized RNFL defects, it appears that OCT has the potential to recognize such defects. Further study is needed to develop new analytical methods that will increase OCT sensitivity for identifying preperimetric localized RNFL damage. (Invest Ophthalmol Vis Sci. 2007;48:1635-1641) DOI:10.1167/iovs.06-0800

$\mathrm{I}_{\mathrm{r}}^{\mathrm{n}}$ n glaucoma, the essential pathologic process is the loss of retinal ganglion cells and their axons. In the clinical setting, glaucoma damage is commonly evaluated by funduscopy, photographs of the optic disc and retinal nerve fiber layer (RNFL), and visual field testing. It has been shown, however, that structural injury precedes visual field loss detectable by stan-

From the ${ }^{1}$ Department of Ophthalmology, Seoul National University College of Medicine, Seoul, Korea; and the ${ }^{2}$ Department of Ophthalmology, Seoul National University Bundang Hospital, Seongnam, Korea.

Supported by a grant from the Seoul National University Bundang Hospital Research Fund.

Submitted for publication July 12, 2006; revised September 4, October 9, and November 26, 2006; accepted February 14, 2007.

Disclosure: T.-W. Kim, None; U.-C. Park, None; K.H. Park, None; D.M. Kim, None

The publication costs of this article were defrayed in part by page charge payment. This article must therefore be marked "advertisement" in accordance with 18 U.S.C. $\$ 1734$ solely to indicate this fact.

Corresponding author: Tae-Woo Kim, Assistant Professor, Department of Ophthalmology, Seoul National University College of Medicine, Seoul National University Bundang Hospital, 300 Gumi-dong, Bundang-gu, Seongnam, Gyunggi-do 463-707, Korea; twkim7@snu.ac.kr. dard automated perimetry (SAP) in many eyes with early glaucomatous optic neuropathy (GON). ${ }^{1-3}$ For example, Sommer et al. ${ }^{4}$ reported that abnormalities of the RNFL were present in $60 \%$ of eyes as much as 6 years before visual field damage was detectable.

Optical coherence tomography (OCT) is a high-resolution, cross-sectional imaging technique that allows in vivo measurement of tissue thickness. ${ }^{5,6}$ The Stratus OCT (Carl Zeiss Meditec, Inc., Dublin, CA) is a third-generation machine that has a resolution of 8 to $10 \mu \mathrm{m}$ and is capable of differentiating between healthy eyes and eyes with glaucoma. ${ }^{7-17}$ Recently, Budenz et al. ${ }^{18}$ reported that the Stratus OCT with its internal normative database showed high sensitivity and specificity for diagnosing glaucoma with manifest visual field (VF) defects. However, there are few reports regarding the use of OCT for detecting glaucomatous damage before detectable changes in SAP.

The purpose of the present study was to evaluate whether retinal nerve fiber layer (RNFL) thickness data generated using Stratus OCT can be used to discriminate between healthy eyes and eyes with a localized RNFL defect (according to red-free fundus photography) but no VF defect (according to standard automated perimetry; SAP).

\section{Methods}

Eyes with localized RNFL defect and normal SAP (preperimetric localized RNFL defective eyes) and healthy control eyes meeting the eligibility criteria were consecutively enrolled from the database of patients who were examined for glaucoma between May 2003 to October 2005 in the Department of Ophthalmology, Seoul National University Bundang Hospital. This study was approved by the Seoul National University Bundang Hospital institutional review board and conformed to the Declaration of Helsinki. Informed consent was obtained from all subjects.

Before the study, all patients underwent a complete ophthalmic examination, including visual acuity, refraction, slit-lamp biomicroscopy, gonioscopy, Goldmann applanation tonometry, dilated stereoscopic examination of the optic disc, red-free fundus photography, OCT, and SAP.

The inclusion criteria were normal SAP results in at least two tests, at least one stereo disc photography assessment, a red-free fundus photography assessment and OCT analysis, all conducted within a 6-month period. Exclusion criteria were a best corrected visual acuity worse than $20 / 40$, a spherical refraction greater than $\pm 5.0 \mathrm{D}$, cylinder correction greater than $\pm 3.0 \mathrm{D}$, closed angles, a history of ocular surgery other than cataract extraction, or disease that may affect the peripapillary area (where OCT measurements are obtained).

Subjects were assigned to either the preperimetric localized RNFL defect group or the healthy control group. Preperimetric localized RNFL defect eyes were defined as those having a localized wedgeshaped RNFL defect clearly visible by red-free photography (a red-free defect) with no glaucomatous VF loss according to SAP. Healthy control eyes were defined as those having normal RNFL configuration according to red-free photography, absence of GON based on the review of stereo disc photographs, intraocular pressure $\leq 21 \mathrm{~mm} \mathrm{Hg}$ 
according to Goldmann applanation tonometry, and normal SAP results. Absence of GON was defined as a cup-to-disc ratio $<0.6$ and an intact neuroretinal rim without peripapillary hemorrhages, notches, or localized pallor.

\section{Visual Field Testing}

Visual field analysis was performed with the Swedish Interactive Threshold Algorithm (SITA) standard, central full-threshold program 30-2, of the Humphrey Field Analyzer II 750 (Carl Zeiss Meditec). Glaucomatous VF loss was defined as a pattern SD outside the $95 \%$ normal limits, or a glaucoma hemifield test result outside normal limits. Only reliable (fixation losses, false positives, and false negatives all $\leq 25 \%)$ VFs were included.

\section{Red-Free RNFL Photography}

Red-free fundus photographs were acquired (EOS D60 digital camera; (Canon, Tochigiken, Japan) after maximum pupil dilation. Sixty degree, wide-angle views of the optic disc, carefully focused on the retina with the built-in split-lines focusing device and centered between the fovea and the optic disc ${ }^{19-21}$ were obtained and reviewed on an LCD monitor. Red-free RNFL photographs were independently evaluated by two of the authors (TWK and UCP) who were masked to the patients clinical information including OCT results and who each classified eyes into one of the following categories: normal, localized defect, diffuse atrophy, or ambiguous (which may have been due to insufficient image quality). Localized RNFL defects according to red-free RNFL photographs were determined when the width at a 1-disc-diameter distance from the edge of the disc was larger than a major retinal vessel, diverged in an arcuate or wedge shape, and reached the edge of the disc. ${ }^{19}$

Only subjects classified by both observers as being either normal or having a localized defect (detected at the same location by both observers) were enrolled in the control and preperimetric RNFL groups, respectively. Subjects diagnosed as having either diffuse atrophy or ambiguous photographs by both observers were classified in the diffuse atrophy or ambiguous group, respectively, and excluded from further analysis. Subjects on whom the two observers disagreed were also classified as ambiguous and excluded from further analysis.

In preperimetric RNFL defect eyes, the localized RNFL defect location was described as a clock hour on the red-free RNFL photograph, which enabled a topographic comparison with OCT-measured RNFL thickness data. For this, the directional angle of the RNFL defect was measured in a way that allows the alignment of OCT images in relation to RNFL photographs. This method has been described elsewhere in detail. $^{20,21}$ In brief, a circle was drawn around the optic nerve head $(\mathrm{ONH})$ on the red-free photograph, the diameter and location of which corresponded as closely as possible to the circle displayed in the video mode of the RNFL thickness analysis report. The two points where the borders of the defect met the circle were then identified, and the directional angles of these border points with respect to the center of the circle were then measured. The directional angle was assessed in a clockwise direction in right eyes and a counterclockwise direction in left eyes, with the temporal equator set at $0^{\circ}$ to correspond to the OCT (Fig. 1A). Defects were assigned a clock hour location according to the measured angles. The angles and corresponding clock hours are shown for both right and left eyes in Figures 1B and 1C. Defect locations are described in terms of the red-free defect clock hour. In cases in which RNFL defects were located over multiple clock hours, all clock hours involving $>3^{\circ}$ over a $360^{\circ}$ circle were included in the red-free clock hour.

\section{Stereoscopic Disc Photography}

Stereoscopic photographs were taken with the digital camera at the same session as red-free fundus photography. Photographs were assessed on an LCD monitor with a stereoscopic viewer (Screen-Vu Stereo Viewer; Berezin Stereo Photography Products, Mission Viejo, CA).

\section{Optical Coherence Tomography}

Subjects were measured with the peripapillary Fast RNFL program of the Stratus OCT (Carl Zeiss Meditec) after a pupillary dilation to a minimum diameter of $5 \mathrm{~mm}$. The imaging lens was positioned $1 \mathrm{~cm}$ from the eye to be examined and was adjusted independently until the retina was in focus. The internal fixation target was used owing to its higher reproducibility. ${ }^{22}$ Data were analyzed with system software (Stratus ver. 3.0 software; Carl Zeiss Meditec). With the Fast RNFL program, the RNFL thickness is determined at 256 points around a set diameter $(3.4 \mathrm{~mm})$ around the center of the optic disc three times during a single scan. These values are averaged to yield 12 clock hour thicknesses, four-quadrant thicknesses, and a global average RNFL thickness measurement $\left(360^{\circ}\right.$ measure). These values are then compared against a normative database of age-matched control subjects to derive percentile values. The four percentile values included in the OCT software are the top 5th percentile, top 95th percentile, bottom 5 th percentile, and bottom percentile.

\section{Data Analysis}

The sensitivity and specificity of OCT parameters were tested by comparison with the OCT's internal normative database. Tested parameters were the global average RNFL thickness at the 5\% and 1\% levels, $\geq 1$ quadrant abnormal at the $5 \%$ and $1 \%$ levels, $\geq 1$ clock hour abnormal at the $5 \%$ and $1 \%$ levels, and all parameters listed on the OCT result printout table (the superior maximum $\left[S_{\max }\right.$, greatest value in the superior quadrant $]$; inferior maximum $\left[I_{\max }\right.$, greatest value in the inferior quadrant]; Max-Min [difference between the greatest and smallest values along the measurement circle $] ; I_{\max } / S_{\max }\left[I_{\max }\right.$-to- $S_{\max }$ ratio]; $S_{\max } / I_{\max } ; S_{\max } / T_{\text {avg }}$ [temporal quadrant average thickness]; $I_{\max } / T_{\text {avg }}$; and $S_{\max } / N_{\text {avg }}$ [nasal quadrant average thickness]) at the $5 \%$

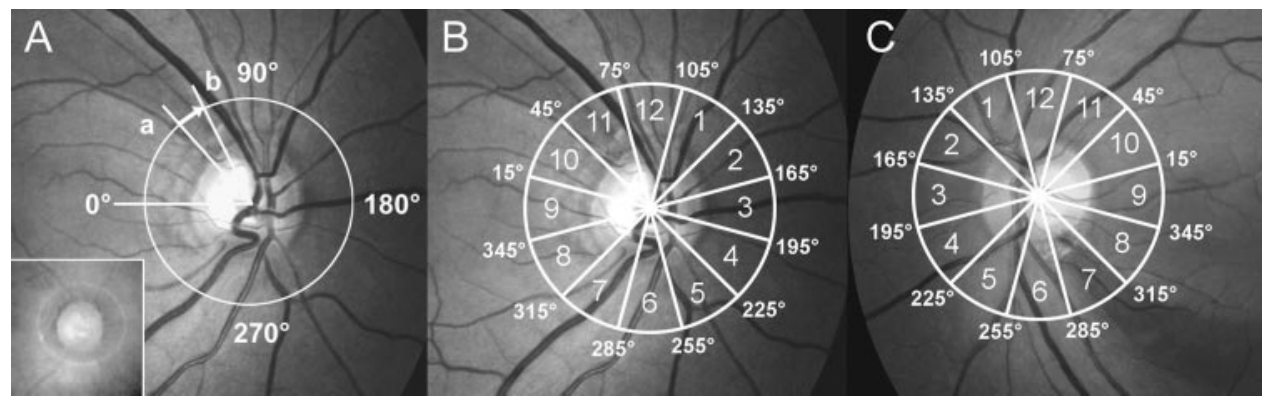

Figure 1. Determination of the clock-hour location of the localized RNFL defect on red-free fundus photographs. (A) A circle was placed around the $\mathrm{ONH}$, the location and diameter of which corresponded as closely as possible to the circle displayed in the video mode of the RNFL thickness analysis report (inset). A reference line was drawn horizontally through the center of the circle. The temporal meeting point of the line with the circle was set at $0^{\circ}$. The two points where the borders of the defect met the circle (points a and b) were identified, and the directional angle of the border points with respect to the center of the circle were measured. The directional angle was assessed in a clockwise direction for right eyes and a counterclockwise direction for left eyes, to correspond to the OCT. Each defect was then assigned a clock-hour position corresponding to the measured angle. In cases where RNFL defects were located over multiple clock hours, all clock hours involving $>3^{\circ}$ over a $360^{\circ}$ circle were included in the red-free clock hour. $(\mathbf{B}, \mathbf{C})$ Circles labeled with angles and corresponding clock hours for both right and left eyes. 
and $1 \%$ levels. Student's $t$-test was used to compare RNFL thicknesses between the two groups. In cases in which both eyes of a patient were eligible for the study, only one eye was randomly chosen for inclusion. When one eye was preperimetric and the other was normal, the preperimetric eye was selected for study.

$P<0.05$ was considered to indicate a significant difference. Statistical analyses were performed with commercial software (JMP 5.1 software; SAS Institute Inc., Cary, NC).

\section{Results}

The study involved the eyes of 195 subjects analyzed with red-free photography, stereo disc photography, SAP-SITA testing (with normal results), and Stratus OCT within a 6-month period. Of these, 41 had diffuse atrophy $(n=21)$ or ambiguous defects $(n=20)$ and were excluded from further analysis. Of the 20 subjects classified as ambiguous, 6 were so classified because of a disagreement between the observers. Disagreement regarding diffuse atrophy versus ambiguity was most common, $(n=3)$ followed by localized defect versus ambiguity $(n=2)$ and diffuse atrophy versus normal $(n=1)$. Although our protocol was set to exclude localized defect cases in which the graders labeled the defect at different locations, no subject was excluded for this reason.

Three eyes classified as having normal RNFL configuration showed glaucomatous disc appearance (cup-to-disc ratio $\geq$ 0.6) according to stereo disc photographs and were also excluded, leaving a sample of 151 eyes of 151 subjects (53 with preperimetric RNFL defects and 98 control subjects). Although the glaucomatous damage was primarily determined by redfree fundus photography, all eyes except four classified in the localized RNFL defect group also showed glaucomatous change within the $\mathrm{ONH}$ according to stereo disc photography. Moreover, the RNFL defect was observed on stereo disc photographs in all eyes. Of the 151 eyes, OCT scans from 11 subjects were deemed unacceptable (i.e., an inadequate signalto-noise ratio $[\leq 33 \mathrm{~dB}]$, or the presence of an uncentered circular ring around the optic disc) and were also excluded from further analyses, leaving a final sample of 140 eyes of 140 patients (49 with preperimetric RNFL defects and 91 control subjects). Of the 91 control eyes, 49 eyes of 49 subjects who were age-matched with subjects with preperimetric RNFL defects were selected for the analysis. The matching for age was performed by randomly selecting one subject with a difference within 2 years of age for each one with preperimetric RNFL defect.

This procedure was performed by one of the authors (UCP) who was masked to the test results. These 49 control eyes were the contralateral eyes of patients with unilateral glaucoma (10 eyes) or those with unilateral suspected glaucoma (39 eyes) identified based on disc appearance.

\section{Patient Characteristics}

All subjects were Korean. There was no significant difference in the mean age between the normal control subjects (55.9 \pm 11.7 years; range, $34-81$ ) and those with preperimetric RNFL defects $(55.8 \pm 11.5$ years; range $34-82)$. No significant difference was found between the two groups in male-to-female ratio or average $\mathrm{VF}$ mean deviation (MD) and pattern standard deviation (PSD; Table 1).

\section{Localized RNFL Defects}

In the preperimetric RNFL defect group, 46 eyes showed 1, 2 eyes showed 2, and 1 eye showed 3 localized RNFL defects according to red-free photographs. Of these 53 RNFL defects, 7 lay over 2 clock hours, resulting in the involvement of 60 clock hours in total. The frequency distribution of red-free defect clock hours is shown in Figure 2. Defects were most commonly observed at 7 o'clock ( 24 subjects) and 11 o'clock (19 subjects). Two eyes had defects at both 7 and 11 o'clock.

\section{Sensitivity and Specificity of OCT Measurements}

The mean values of Stratus OCT RNFL parameters for each group are shown in Table 2 . The preperimetric RNFL defect and normal groups differed in terms of the mean parametric global average thickness values, the temporal and inferior thicknesses, and clock hour thicknesses at 7, 8, 9, 10 and 11 o'clock $(P<0.05)$.

The sensitivity of the various Stratus OCT RNFL thickness parameters ranged from $2.0 \%$ to $40.8 \%$ (Table 3 ). The highest sensitivity $(40.8 \%, 20 / 49$ eyes) was yielded with the criterion of $\geq 1$ clock hour abnormal at the $5 \%$ level and had a specificity of $85.7 \%$. Other parameters showed lower sensitivities either at the $5 \%$ or $1 \%$ level with some increase in specificity.

\section{Topographic Relationship between OCT-Measured Clock-Hour RNFL Thickness and the Red-Free RNFL Defect}

Of the 20 eyes with an OCT abnormality of $\geq 1$ clock hour at the 5\% level, 15 (75\%) showed abnormalities at the same clock hour in both OCT and red-free photographs. The remaining five (25\%) eyes showed an OCT abnormality within 1 clock hour of the red-free clock hour.

A subset of 21 eyes with a single localized RNFL defect at 7 o'clock in the red-free fundus photography showed thinner OCT-measured RNFL thicknesses at 5, 6, 7 and 8 o'clock compared with 49 control eyes, with the 7 o'clock defect being the most significantly different (Fig. 3A). Of these 21 subset eyes, 3 had RNFL defects at both 7 and 8 o'clock, and 1 had defects at both 6 and 7 o'clock in the red-free fundus photography. For the remaining 17 eyes, the RNFL defect was limited to within the 7 o'clock sector. Similarly, a subset of 17 eyes having a single localized RNFL defect at 11 o'clock in the red-free fundus photograph showed thinner OCT-measured RNFL thicknesses in the 9, 10 and 11 o'clock sectors compared with normal control eyes (Fig. 3B). Of these 17 subset eyes, 1 had an RNFL defect involving both 10 and 11 o'clock. For the remaining 16 eyes, the RNFL defect was limited to within the 11 o'clock sector in the red-free fundus photograph. Separate analysis was not performed on other subsets of subjects, since the number of subjects with red-free defects at other than 7 and 11 o'clock was too small for meaningful statistical analysis.

\section{Discussion}

The present study showed that of the multiple parameters appearing on current Stratus OCT printouts, none was sensitive enough to identify preperimetric localized RNFL damage. The highest sensitivity was only $40.8 \%$ and was achieved using

TABLE 1. Demographic Characteristics of Study Subjects

\begin{tabular}{lccc}
\hline & $\begin{array}{c}\text { Preperimetric } \\
\text { RNFL Defect } \\
(\boldsymbol{n}=49)\end{array}$ & $\begin{array}{c}\text { Control } \\
(\boldsymbol{n}=49)\end{array}$ & $\boldsymbol{P}$ \\
\hline Age (y) & $55.8 \pm 11.5$ & $55.9 \pm 11.7$ & $0.945^{*}$ \\
Male/female & $26 / 23$ & $26 / 23$ & $1.000 \dagger$ \\
MD (dB) & $0.09 \pm 1.56$ & $-0.09 \pm 1.78$ & $0.599^{*}$ \\
PSD (dB) & $2.03 \pm 0.54$ & $1.99 \pm 0.55$ & $0.703^{*}$ \\
\hline
\end{tabular}

\footnotetext{
* $t$-test.
}

$\dagger \chi^{2}$ test. 


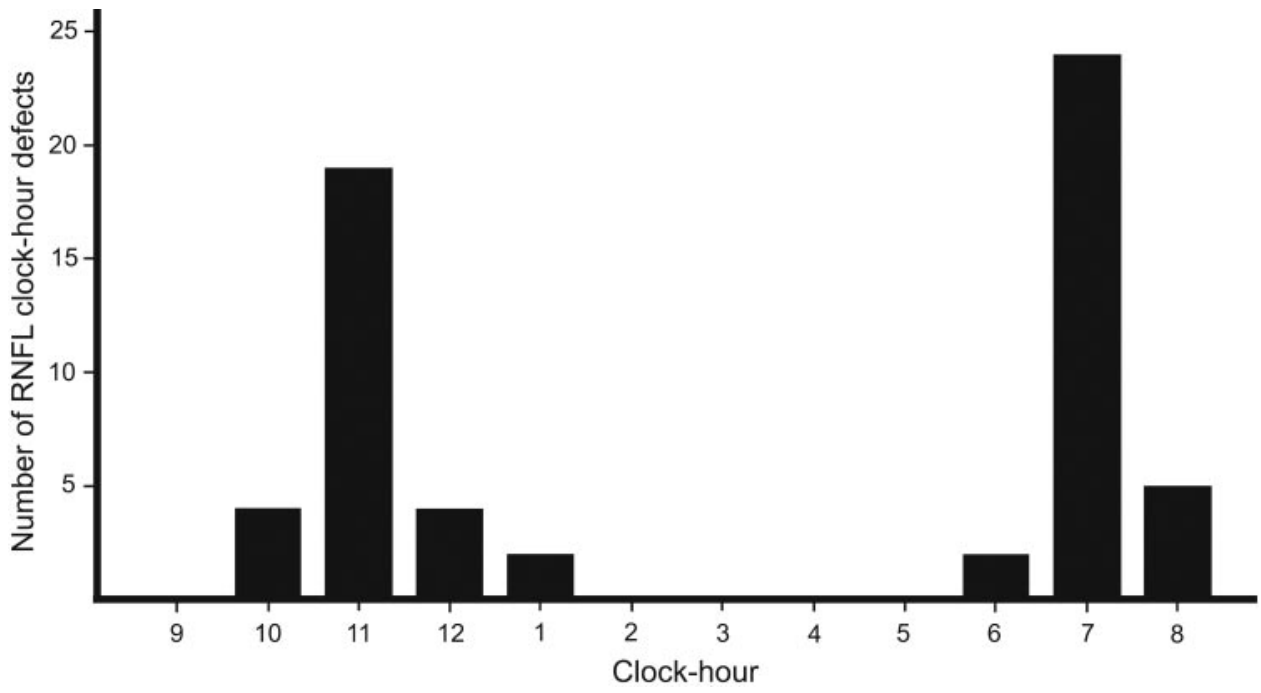

FIGURE 2. Frequency distribution of localized RNFL defects in terms of clock-hour positions after red-free RNFL photography of 49 eyes with preperimetric RNFL defect. the parameter of $\geq 1$ clock hour abnormal at the $<5 \%$ level. This result is in contrast with previous studies on the performance of OCT for detecting glaucoma with manifest VF defects. Using the same criterion ( $\geq 1$ clock hour abnormal at the $<5 \%$ level), Budenz et al. ${ }^{18}$ obtained a sensitivity of $89 \%$ and a specificity of $92 \%$ for glaucoma with manifest VF defects. In addition, a study performed by our group demonstrated a sensitivity of $85.9 \%$ and a specificity of $97.4 \%$ for detecting localized RNFL defects accompanied by corresponding VF defects. ${ }^{21}$ The reason the present study showed a lower sensitivity of OCT for preperimetric RNFL defects may be that he RNFL damage was less marked than in previous investigations in which the study eyes had manifest VF defects.

Another possible reason for the low sensitivity of OCT in the present study is ethnic differences between the sample population of the Stratus OCT intrinsic normative database and the study subjects. The current intrinsic normative database was derived from a measurement of 328 normal subjects, comprising 205 (63\%) white, 79 (24\%) Hispanic, 27 (8\%) black, and only 11 (3\%) Asian people. ${ }^{23}$ In contrast, all study patients were Korean. Given that optic disc parameters ${ }^{24,25}$ and RNFL thickness ${ }^{26}$ can vary according to race/ethnicity, it may be that the absolute RNFL thickness magnitude in Koreans differs from

TABLe 2. RNFL Thickness in Preperimetric RNFL Defect and Control Subjects

\begin{tabular}{|c|c|c|c|}
\hline Location & $\begin{array}{l}\text { Preperimetric } \\
\text { RNFL Defect } \\
(n=49)\end{array}$ & $\begin{array}{c}\text { Control } \\
(n=49)\end{array}$ & $\boldsymbol{P}$ \\
\hline 1 o'clock & $111.0 \pm 27.3$ & $113.0 \pm 23.2$ & 0.691 \\
\hline 2 o'clock & $82.9 \pm 16.9$ & $87.4 \pm 18.0$ & 0.205 \\
\hline 3 o'clock & $61.2 \pm 14.4$ & $65.2 \pm 14.5$ & 0.179 \\
\hline 4 o'clock & $73.1 \pm 16.0$ & $76.8 \pm 16.6$ & 0.262 \\
\hline 5 o'clock & $105.0 \pm 21.0$ & $111.4 \pm 19.5$ & 0.121 \\
\hline 6 o'clock & $124.9 \pm 27.4$ & $134.3 \pm 27.1$ & 0.091 \\
\hline 7 o'clock & $115.4 \pm 29.0$ & $140.1 \pm 18.7$ & $<0.001$ \\
\hline 8 o'clock & $71.2 \pm 12.8$ & $80.9 \pm 14.2$ & $<0.001$ \\
\hline 9 o'clock & $57.7 \pm 9.1$ & $62.5 \pm 9.2$ & 0.011 \\
\hline 10 o'clock & $82.2 \pm 15.5$ & $90.4 \pm 16.3$ & 0.013 \\
\hline 11 o'clock & $119.0 \pm 23.3$ & $134.5 \pm 21.9$ & 0.001 \\
\hline 12 o'clock & $120.1 \pm 31.0$ & $124.1 \pm 21.9$ & 0.454 \\
\hline Superior quadrant & $116.7 \pm 3.0$ & $123.8 \pm 16.7$ & 0.083 \\
\hline Nasal quadrant & $72.3 \pm 14.2$ & $76.4 \pm 14.9$ & 0.162 \\
\hline Inferior quadrant & $115.0 \pm 21.5$ & $128.6 \pm 17.9$ & 0.001 \\
\hline Temporal quadrant & $70.9 \pm 10.9$ & $77.8 \pm 11.0$ & 0.002 \\
\hline Average RNFL thickness & $93.6 \pm 13.4$ & $101.7 \pm 10.9$ & 0.002 \\
\hline
\end{tabular}

that in other ethnic populations. Such a difference would affect the diagnostic accuracy of the current Stratus OCT machine when analyzing the Korean population.

Several studies have shown that OCT may be better at detecting glaucoma with VF defects than suspected glaucoma. Kanamori et al. ${ }^{11}$ compared OCT findings in normal eyes, eyes with ocular hypertension, eyes with suspected glaucoma (glaucomatous optic disc with normal automated VF), and eyes with glaucoma. In that study, the highest sensitivity for the glaucoma suspect group was $53 \%$ when the specificity was set at $90 \%$, whereas a sensitivity of $74 \%$ at a specificity of $91 \%$ was observed in the early perimetric glaucoma group based on RNFL thickness in the inferior quadrant. Nouri-Mahdavi et al. ${ }^{27}$ and Leung et al. ${ }^{10}$ reported that OCT was less able to discriminate between those with suspected glaucoma suspects and normal subjects compared with discriminating between perimetric glaucoma patients and normal subjects. However, in those studies previous versions of OCT were used, ${ }^{11,27}$ or the studies were conducted before the use of internal normative data. ${ }^{10,11,27}$ In contrast, the present study evaluated the diagnostic ability of the current Stratus OCT version, with the measured value compared against the internal normative database. To our knowledge, this is the first report on the perfor-

TABLE 3. Sensitivity and Specificity of OCT RNFL Thickness Parameters for Diagnosis of Preperimetric RNFL Defect

\begin{tabular}{|c|c|c|}
\hline OCT Parameter & $\begin{array}{c}\text { Sensitivity } \\
(\%)\end{array}$ & $\begin{array}{c}\text { Specificity } \\
(\%)\end{array}$ \\
\hline $\begin{array}{l}\geq 1 \text { Clock hours abnormal at the } 5 \% \\
\text { level }\end{array}$ & 40.8 & 85.7 \\
\hline \multicolumn{3}{|l|}{$\geq 1$ Clock hours abnormal at the $1 \%$} \\
\hline level & 16.3 & 96.7 \\
\hline$\geq 1$ Quadrants abnormal at the $5 \%$ level & 26.5 & 93.4 \\
\hline$\geq 1$ Quadrants abnormal at the $1 \%$ level & 10.2 & 98.9 \\
\hline$I_{\max } / S_{\max }$ abnormal at the $5 \%$ level & 26.5 & 91.2 \\
\hline$S_{\max } / I_{\max }$ abnormal at the $5 \%$ level & 6.1 & 93.4 \\
\hline$S_{\max } / T_{\text {avg }}$ abnormal at the $5 \%$ level & 16.3 & 89.0 \\
\hline$I_{\max } / T_{\text {avg }}$ abnormal at the $5 \%$ level & 20.4 & 87.9 \\
\hline$S_{\max } / N_{\text {avg }}$ abnormal at the $5 \%$ level & 2.0 & 98.9 \\
\hline Max-min abnormal at the $5 \%$ level & 22.5 & 89.0 \\
\hline$S_{\max }$ abnormal at the $5 \%$ level & 16.3 & 96.7 \\
\hline$I_{\max }$ abnormal at the $5 \%$ level & 32.7 & 97.8 \\
\hline \multicolumn{3}{|l|}{ Average RNFL thickness abnormal at the } \\
\hline $5 \%$ level & 14.3 & 98.9 \\
\hline \multicolumn{3}{|l|}{ Average RNFL thickness abnormal at the } \\
\hline $1 \%$ level & 4.1 & 100 \\
\hline
\end{tabular}



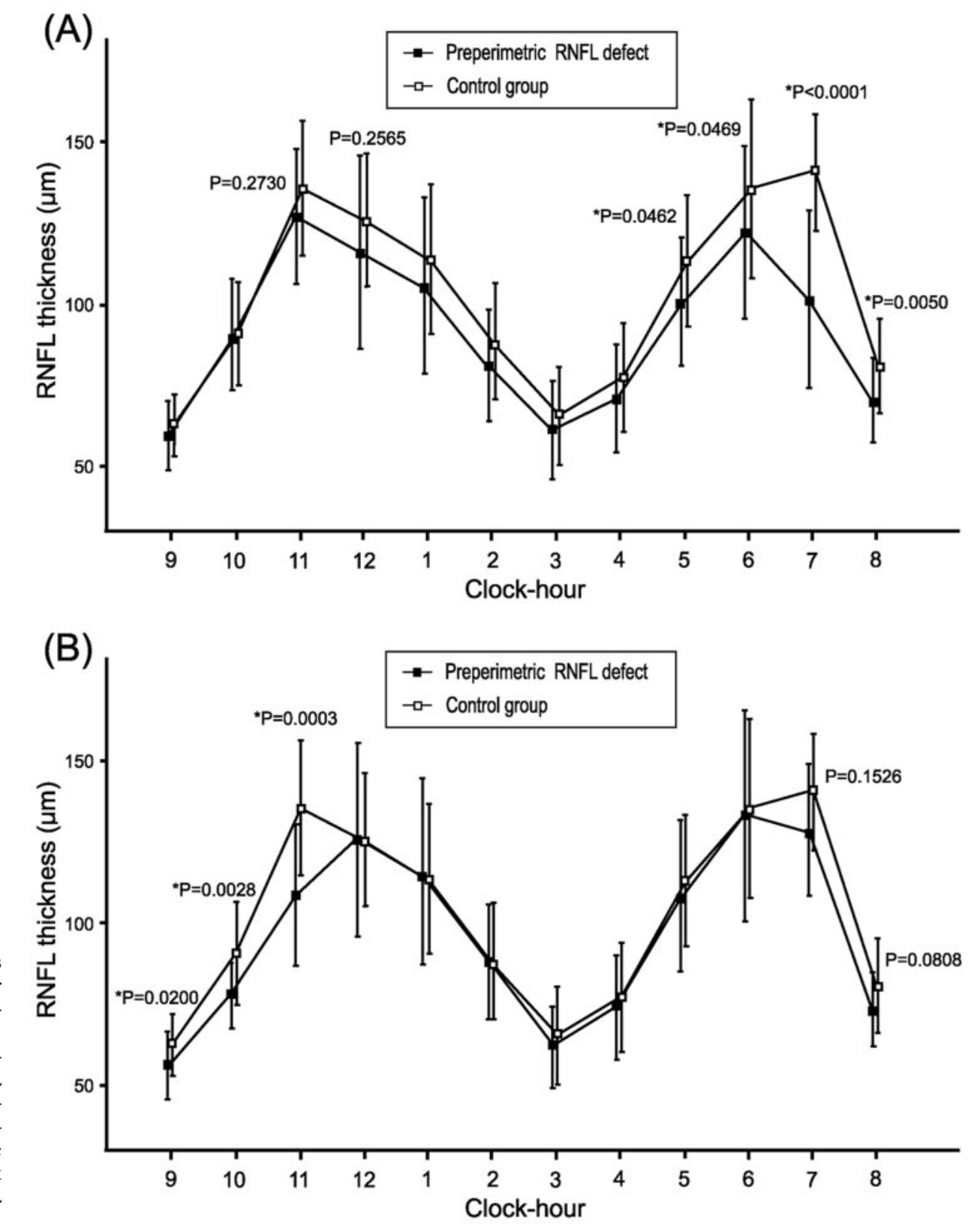

FiguRE 3. Comparison of Stratus OCT clock-hour retinal nerve fiber layer (RNFL) thickness profiles between 49 healthy eyes and (A) 21 eyes with preperimetric RNFL defects having a single localized RNFL defect involving the 7-o'clock position and (B) 17 eyes with preperimetric RNFL defects having a single RNFL defect involving the 11-o'clock position, according to red-free RNFL photography. *Significant differences.

mance of the Stratus OCT with its normative database in patients with preperimetric RNFL defects.

Although we found that the sensitivity of the Stratus OCT with the normative database was not satisfactory, subgroup analyses of eyes with an RNFL defect in a given clock hour (e.g., 7 or 11 o'clock) demonstrated that the sector with the most significantly deviated OCT-measured RNFL thickness corresponded to that identified by red-free fundus photography when compared with thickness in healthy control subjects. These data suggest that Stratus OCT may recognize localized nerve fiber layer defects before the development of a VF defect. For this capability to be clinically useful, further study appears necessary to develop new analysis methods or parameters to increase the sensitivity of OCT for diagnosis of preperimetric glaucoma.

In subset analyses, the subgroup with a single red-free defect at 7 o'clock showed a tendency toward reduced RNFL thickness at 11 and 12 o'clock, and vice versa. This result is consistent with previous observations that RNFL change is commonly observed in the upper and lower quadrants of eyes with VF defects confined to one hemifield, ${ }^{28}$ and further suggests that RNFL changes may exist in apparently normal quadrants even at the preperimetric stage.

In the present study, eyes were primarily classified according to red-free photography data, since RNFL examination may provide more information about minor loss of axons than evaluation of the $\mathrm{ONH}$ where axons are densely packed. ${ }^{29}$ Although localized RNFL defects are easily identified in red-free photography, it is sometimes difficult to discriminate between normal eyes and those with diffuse atrophy. ${ }^{30}$ Hence, we excluded eyes with diffuse RNFL atrophy from the present study, and eyes classified as having a normal RNFL configuration were assessed twice in a masked review of stereoscopic disc photographs. Furthermore, by including only eyes with localized RNFL defects, in which the border of the defect could be clearly defined, we were able to evaluate the topographic relationship between the abnormal RNFL thickness location according to OCT and the red-free RNFL defect location. 
The design of the present study, which included only eyes with localized defects, resulted only in information regarding the ability of OCT to detect localized RNFL defects. However, glaucoma may appear as diffuse RNFL atrophy or without a prominent RNFL defect. The merit of OCT for detecting this type of abnormality has yet to be determined. Further studies are needed to determine the performance of OCT in those types of damage. To assess the performance of OCT in cases that are ambiguous based on conventional technologies, studies with a prospective, longitudinal design may be necessary because there is no reference standard that can be used to determine definitively the true nature of such cases in a single examination.

Several recent studies ${ }^{31,32}$ have shown that OCT diagnostic performance may be influenced by the size of the optic disc. This influence may be explained by variations in the OCTmeasured RNFL thickness depending on the disc size. ${ }^{32,33}$ Because it is likely that variables influencing the diagnostic performance of imaging tests would have more influence in the early compared with the advanced stage of disease, it may be that such influence was substantial in the present study, contributing, at least in part, to the low sensitivity observed. It seems necessary to evaluate whether correcting the effect of disc size (for example by customizing the diameter of the scan according to ONH size) may increase the OCT diagnostic performance, especially in patients with early-stage glaucoma.

In the present study, healthy control eyes were from patients with unilateral glaucoma or suspected glaucoma. Although those eyes showed normal findings in both structural and functional assessments, it is possible that they had subclinical glaucomatous damage, because primary open-angle glaucoma is generally a bilateral disease, albeit the time frame may be asymmetric. However, this possibility does not invalidate the findings of the present study. On the contrary, it reinforces the ability of quantitative OCT analysis to identify abnormalities, even when the normal group may not be completely free of glaucomatous damage.

In summary, based on the normative database for preperimetric localized RNFL defects, the current Stratus OCT RNFL thickness parameters showed poor sensitivity. However, it appears that OCT may have the potential to recognize preperimetric RNFL defects. Further study is necessary to develop new analytical methods for increasing OCT sensitivity to detect preperimetric RNFL defects.

\section{References}

1. Katz J, Sommer A, Gaasterland DE, Anderson DR. Comparison of analytic algorithms for detecting glaucomatous visual field loss. Arch Ophthalmol. 1991;109:1684-1689.

2. Tuulonen A, Lehtola J, Airaksinen PJ. Nerve fiber layer defects with normal visual fields: do normal optic disc and normal visual field indicate absence of glaucomatous abnormality? Opbthalmology. 1993;100:587-597; discussion 597-588.

3. Quigley HA, Katz J, Derick RJ, Gilbert D, Sommer A. An evaluation of optic disc and nerve fiber layer examinations in monitoring progression of early glaucoma damage. Opbthalmology. 1992;99: $19-28$.

4. Sommer A, Katz J, Quigley HA, et al. Clinically detectable nerve fiber atrophy precedes the onset of glaucomatous field loss. Arch Ophthalmol. 1991;109:77-83.

5. Huang D, Swanson EA, Lin CP, et al. Optical coherence tomography. Science. 1991;254:1178-1181.

6. Hee MR, Izatt JA, Swanson EA, et al. Optical coherence tomography of the human retina. Arch Ophthalmol. 1995;113:325-332.

7. Mistlberger A, Liebmann JM, Greenfield DS, et al. Heidelberg retina tomography and optical coherence tomography in normal, ocularhypertensive, and glaucomatous eyes. Opbthalmology. 1999;106: 2027-2032.
8. Medeiros FA, Zangwill LM, Bowd C, et al. Evaluation of retinal nerve fiber layer, optic nerve head, and macular thickness measurements for glaucoma detection using optical coherence tomography. Am J Opbthalmol. 2005;139:44-55.

9. Wollstein G, Ishikawa H, Wang J, Beaton SA, Schuman JS. Comparison of three optical coherence tomography scanning areas for detection of glaucomatous damage. Am J Opbthalmol. 2005;139: $39-43$.

10. Leung CK, Chan WM, Yung WH, et al. Comparison of macular and peripapillary measurements for the detection of glaucoma: an optical coherence tomography study. Ophthalmology. 2005;112: $391-400$.

11. Kanamori A, Nakamura M, Escano MF, et al. Evaluation of the glaucomatous damage on retinal nerve fiber layer thickness measured by optical coherence tomography. Am J Opbthalmol. 2003; 135:513-520

12. Schuman JS, Hee MR, Arya AV, et al. Optical coherence tomography: a new tool for glaucoma diagnosis. Curr Opin $O p b$ thalmol. 1995;6:89-95.

13. Schuman JS, Hee MR, Puliafito CA, et al. Quantification of nerve fiber layer thickness in normal and glaucomatous eyes using optical coherence tomography. Arch Opbthalmol. 1995;113: 586-596.

14. Bowd C, Weinreb RN, Williams JM, Zangwill LM. The retinal nerve fiber layer thickness in ocular hypertensive, normal, and glaucomatous eyes with optical coherence tomography. Arch Ophthalmol. 2000;118:22-26.

15. Hoh ST, Greenfield DS, Mistlberger A, et al. Optical coherence tomography and scanning laser polarimetry in normal, ocular hypertensive, and glaucomatous eyes. Am J Ophthalmol. 2000;129: $129-135$

16. Zangwill LM, Williams J, Berry CC, Knauer S, Weinreb RN. A comparison of optical coherence tomography and retinal nerve fiber layer photography for detection of nerve fiber layer damage in glaucoma. Opbthalmology. 2000;107:1309-1315.

17. Zangwill LM, Bowd C, Berry CC, et al. Discriminating between normal and glaucomatous eyes using the Heidelberg Retina Tomograph, GDx Nerve Fiber Analyzer, and Optical Coherence Tomograph. Arch Opbthalmol. 2001;119:985-993.

18. Budenz DL, Michael A, Chang RT, McSoley J, Katz J. Sensitivity and specificity of the StratusOCT for perimetric glaucoma. Ophthalmology. 2005;112:3-9.

19. Hoyt WF, Frisen L, Newman NM. Fundoscopy of nerve fiber layer defects in glaucoma. Invest Opbthalmol. 1973;12:814-829.

20. Hwang JM, Kim TW, Park KH, Kim DM, Kim H. Correlation between topographic profiles of localized retinal nerve fiber layer defects as determined by optical coherence tomography and red-free fundus photography. J Glaucoma. 2006;15:223228.

21. Jeoung JW, Park KH, Kim TW, Khwarg SI, Kim DM. Diagnostic ability of optical coherence tomography with a normative database to detect localized retinal nerve fiber layer defects. Ophthalmology. 2005;112:2157-2163.

22. Schuman JS, Pedut-Kloizman T, Hertzmark E, et al. Reproducibility of nerve fiber layer thickness measurements using optical coherence tomography. Ophthalmology. 1996;103:1889-1898.

23. Patella VM. StratusOCT: Establisbment of Normative Reference Values for Retinal Nerve Fiber Layer Thickness Measurements. Dublin CA: Carl Zeiss Meditec, Inc.; 2003.

24. Varma R, Tielsch JM, Quigley HA, et al. Race-, age-, gender-, and refractive error-related differences in the normal optic disc. Arch Ophthalmol. 1994;112:1068-1076.

25. Chi T, Ritch R, Stickler D, et al. Racial differences in optic nerve head parameters. Arch Ophthalmol. 1989;107:836-839.

26. Tjon-Fo-Sang MJ, Lemij HG. Retinal nerve fiber layer measurements in normal black subjects as determined with scanning laser polarimetry. Ophthalmology. 1998;105:78-81.

27. Nouri-Mahdavi K, Hoffman D, Tannenbaum DP, Law SK, Caprioli J. Identifying early glaucoma with optical coherence tomography. Am J Opbthalmol. 2004;137:228-235.

28. Kook MS, Lee SU, Sung KR, et al. Pattern of retinal nerve fiber layer damage in Korean eyes with normal-tension glaucoma and hemi- 
field visual field defect. Graefes Arch Clin Exp Ophthalmol. 2002; 240:448-456.

29. Teesalu P, Tuulonen A, Airaksinen PJ. Optical coherence tomography and localized defects of the retinal nerve fiber layer. Acta Ophthalmol Scand. 2000;78:49-52.

30. Airaksinen PJ, Tuulonen A, Werner EB. Clinical evaluation of the optic disc and retinal nerve fiber layer. In: Ritch R, Shields MB, Krupin T, eds. The Glaucomas. St. Louis: Mosby; 1996:617-657.

31. Medeiros FA, Zangwill LM, Bowd C, Sample PA, Weinreb RN. Influence of disease severity and optic disc size on the diagnostic performance of imaging instruments in glaucoma. Invest Ophthalmol Vis Sci. 2006;47:1008-1015.

32. Savini G, Zanini M, Carelli V, et al. Correlation between retinal nerve fibre layer thickness and optic nerve head size: an optical coherence tomography study. Br J Ophthalmol. 2005;89:489492.

33. Bowd C, Zangwill LM, Blumenthal EZ, et al. Imaging of the optic disc and retinal nerve fiber layer: the effects of age, optic disc area, refractive error, and gender. J Opt Soc Am A Opt Image Sci Vis. 2002;19:197-207. 